\title{
Factores de riesgo sociales y ambientales relacionados con casos de leptospirosis de manejo ambulatorio y hospitalario, Turbo, Colombia
}

\author{
Dionisia Yusti', Margarita Arboleda², Piedad Agudelo-Flórez² \\ 1 División de Salud Pública, Facultad de Medicina, Universidad CES, Medellín, Colombia \\ 2 Instituto Colombiano de Medicina Tropical, Universidad CES, Medellín, Colombia \\ Institución donde se realizó el trabajo: Instituto Colombiano de Medicina Tropical, Universidad CES, Medellín, \\ Colombia
}

Introducción. La leptospirosis es un problema de salud pública en el Urabá colombiano y poco se sabe sobre las condiciones ambientales y sociales de esta enfermedad en la región.

Objetivo. Explorar algunos factores de riesgo relacionados con leptospirosis de manejo hospitalario y ambulatorio, en pacientes del municipio de Turbo durante los años 2010 y 2011.

Materiales y métodos. Mediante un estudio descriptivo se exploraron factores relacionados con la exposición a Leptospira spp. (aspectos socio-demográficos, hábitos, condiciones físicas y de saneamiento de la vivienda, hacinamiento, fuentes de agua potable, presencia de roedores sinantrópicos y convivencia con animales) en pacientes con leptospirosis que requirieron manejo hospitalario en el municipio de Turbo durante los años 2010 y 2011. Se utilizaron medidas estadísticas estandarizadas para estudios descriptivos.

Resultados. Se encontró que el hábito de caminar descalzo en ambientes domésticos representó $4,27(1,32-13,82)$ veces el riesgo para presentar leptospirosis de manejo hospitalario $(p=0,012)$. El análisis multivariado exploratorio mostró que la presencia de fauna silvestre en las viviendas puede estar relacionada también con casos de manejo hospitalario. Este hallazgo representó 4,22 (1,1315,72 ) veces el riesgo comparado con los casos ambulatorios que manifestaron no tener este tipo de animales dentro de la vivienda $(p=0,032)$.

Conclusión. Este estudio plantea bases para diseñar e implementar intervenciones efectivas, orientadas desde el perfil de riesgos al que se exponen sus habitantes, en un área geográfica que exhibe una epidemiología dinámica de contexto complejo para leptospirosis.

Palabras clave: leptospirosis, factores de riesgo, epidemiología, animales salvajes

doi: http://dx.doi.org/10.7705/biomedica.v33i0.1457

Social and environmental risk factors associated with leptospirosis of inpatient and outpatient management, Turbo, Colombia

Introduction: Leptospirosis is a public health problem in the Colombian Urabá area and little is known about the environmental and social conditions of this disease in the region.

Objective: To explore some risk factors associated with leptospirosis of inpatient and outpatient management in the municipality of Turbo during the years 2010-2011.

Materials and methods: A descriptive study was performed to explore factors related to Leptospira spp. exposure (socio-demographic aspects, habits, housing physical and sanitary conditions, overcrowding, drinking water sources, presence of synanthropic rodents, and living with animals) in patients with leptospirosis that required hospital management in the municipality of Turbo during the years 2010 and 2011. We used standard statistical measures for descriptive studies.

Results: We found that the habit of barefoot walking in domestic environments represented 4.27 (1.32 to 13.82) times the risk for leptospirosis present in inpatient management $(p=0.012)$. Exploratory multivariate analysis showed that the presence of wildlife in homes could also be related to cases of inpatient management. This finding represented 4.22 (1.13 to 15.72) times the risk compared with outpatient cases reported as not having this type of animals inside their home $(p=0.032)$.

Conclusion: This study suggests a basis for designing and implementing effective interventions,

\section{Contribución de los autores:}

Dionisia Yusti: diseño del estudio, recolección y análisis de la información.

Margarita Arboleda: análisis de la información y revisión del manuscrito.

Piedad Agudelo-Flórez: diseño del estudio, análisis de la información, edición y revisión del manuscrito. 
thought from the risk profile its inhabitants are exposed to, in a geographic area that exhibits a dynamic epidemiology of complex leptospirosis context.

Key words: Leptospirosis, risk factors, epidemiology, wild animals. doi: http://dx.doi.org/10.7705/biomedica.v33i0.1457

La leptospirosis es una enfermedad de carácter zoonótico, considerada como un problema de salud pública a nivel mundial, particularmente en áreas tropicales y subtropicales de países en desarrollo. La enfermedad es causada por las especies patógenas del género Leptospira que se alojan en los túbulos renales de los huéspedes adaptados y vulnerables, siendo arrojadas al ambiente donde pueden sobrevivir por semanas o meses (1). Esta zoonosis ocasiona pérdidas económicas a la producción pecuaria mundial, principalmente de ganado porcino y bovino, al causar falla reproductiva en los huéspedes crónicos o de mantenimiento, pero también afecta en forma grave a más de un millón de personas anualmente en el mundo quienes se consideran huéspedes vulnerables de Leptospira spp. $(1,2)$.

Para los humanos, la leptospirosis es una enfermedad potencialmente mortal pero que puede tratarse con antibióticos (2); su espectro clínico va desde la enfermedad asintomática, pasando incluso por síntomas leves similares a una influenza, hasta asemejar hepatitis, salmonelosis, dengue, fiebres hemorrágicas virales, rickettsiosis y meningitis. Puede cursar en su forma grave como un síndrome icterohemorrágico con compromiso renal y multisistémico, en ocasiones fatal, o como un cuadro de hemorragia pulmonar (3). Al ser frecuente en zonas tropicales endémicas para otras infecciones, como el dengue, su cuadro clínico genera confusión para el personal de la salud, siendo generalmente subdiagnosticada y, por ende, subnotificada.

A nivel mundial, la magnitud en ascenso de este problema de salud pública se atribuye a las condiciones climáticas y ambientales, que favorecen la perpetuación de la bacteria en el ambiente, y a las migraciones de animales y personas hacia zonas urbanas y periurbanas de los grandes centros poblados donde las deficientes condiciones de saneamiento básico determinan la exposición a la orina de animales infectados con Leptospira

Correspondencia:

Piedad Agudelo-Flórez, Carrera 43A N 52 Sur-99, Sabaneta, Colombia.

Teléfono: (574) 305 3500, extensión 2314; fax: (574) 3014258

pagudelo@ces.edu.co

Recibido: 17/11/12; aceptado:02/04/13 spp. (4,5). En Colombia, la notificación de casos y muertes por leptospirosis al Instituto Nacional de Salud, mediante el Sistema de Vigilancia en Salud Pública (Sivigila), se inició de manera constante y con tendencia al aumento en el 2008. En ese año, se notificaron 1.357 casos y 29 muertes; en el 2009 fueron 1.816 casos y 31 muertes; en el 2010, el número de casos ascendió a 2.261 y 57 muertes, con un comportamiento similar en 2011 cuando se notificaron 2.478 casos y 56 muertes (6). El aumento en la notificación se puede explicar por la implementación de la vigilancia en salud pública para leptospirosis y por el período invernal asociado con el fenómeno climático de La Niña que se presentó en el país desde el año 2009.

Una de las regiones colombianas que se reconoce como de frecuente transmisión de leptospirosis es el departamento de Antioquia, donde se registraron 341 casos confirmados en el 2011, lo cual supone una tasa de 5,55 por 100.000 habitantes y una letalidad del $1,17 \%$ con 4 pacientes fallecidos por esta causa. La subregión del departamento de donde procede la mayoría de estos casos es la zona noroccidental del Litoral Caribe, conocida como la región de Urabá; esta se ha caracterizado por migración constante de la población en busca de fuentes de trabajo, por la presentación de problemas de orden público y por inundaciones en épocas de lluvias. De los casos de leptospirosis notificados y confirmados en Colombia a través del Sivigila, durante el 2011, el 5,65\% (140/2.478) procedía de la región de Urabá.

Se dispone de pocos estudios que hayan explorado los factores de riesgo que se relacionan con la exposición a Leptospira spp. en población de la zona. El primero de ellos fue llevado a cabo en las zonas urbanas de nueve municipios del Urabá antioqueño, registrándose una seroprevalencia general de 12,5 $\%$ (74/591), $\left(\mathrm{IC}_{95 \%}: 10,0-15,4\right)$ para la región. En seis de los nueve municipios muestreados se encontraron anticuerpos contra Leptospira spp. en la población estudiada, siendo Carepa (27,3\%), Necoclí (25\%) y San Pedro de Urabá (25\%) los de mayor porcentaje de seroprevalencia, seguidos por Apartadó (14,8\%), Turbo (11,8\%) y Chigorodó (7,5\%). No se observaron diferencias estadísticamente significativas en los casos seropositivos por edad, sexo, raza, oficio, años de residencia en la zona y vivienda de construcción adecuada o inadecuada. En todos los grupos de edad 
se encontraron personas con anticuerpos contra, al menos, uno de los seis serogrupos de Leptospira spp. evaluados, principalmente Icteroahemorragiae y Grippotyphosa (7).

El segundo estudio se llevó a cabo en el municipio de Necoclí, donde previamente se había documentado un brote de rickettsiosis asociado con otras infecciones endémicas, entre ellas leptospirosis. El estudio incluyó a 642 habitantes de la zona rural del municipio y registró una seroprevalencia de 35,6 \% principalmente dirigida a los serogrupos Icteroahemorragiae y Hardjo. Como en el anterior estudio, no se presentó asociación entre los casos seropositivos y factores demográficos como edad, sexo y ocupación, pero sí se encontró asociación con habitar en un área con cobertura vegetal mayor al $10 \%$ y con habitar en viviendas que tenían almacenamiento de cosechas sin cerramiento completo (8).

Estos reportes de prevalencia de leptospirosis para la zona de Urabá, la determinación de algunos factores de riesgo y el aumento en la notificación de casos confirmados y de casos fatales procedentes de sus municipios, ameritan explorar las condiciones ambientales y sociales del entorno donde residen las personas a quienes se les ha diagnosticado leptospirosis, con el fin de contribuir al diseño e implementación de intervenciones efectivas y específicas para esta zona geográfica. En este sentido, se realizó el presente estudio en el municipio de Turbo, con el objetivo de caracterizar algunos aspectos sociodemográficos, habitacionales y del entorno de un grupo de pacientes con leptospirosis, manejados tanto ambulatoria como hospitalariamente.

\section{Materiales y métodos}

\section{Área y población de estudio}

La región del Urabá antioqueño está localizada al noroeste del departamento de Antioquia, Colombia, en el Golfo de Urabá, y cuenta con 11 municipios (figura 1a), entre ellos Turbo, el más

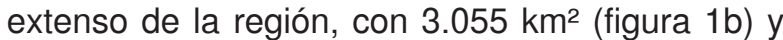
una población de 147.243 habitantes para el año 2012 (Departamento Administrativo Nacional de Estadística, DANE. Proyecciones Censo 2005). Más de la mitad de sus habitantes $(70,12 \%)$ vive en condición de pobreza y, de estos, el $33 \%$ se encuentra en condiciones de miseria (DANE, boletín censo general 2005).

La población de referencia del presente estudio correspondió a los casos febriles que consultaron a las instituciones de salud del municipio de Turbo, durante los años 2010 y 2011. Por su parte, la población de estudio fueron los casos febriles agudos con diagnóstico de leptospirosis.

\section{Tipo de estudio, instrumentos y procedimientos}

El presente estudio descriptivo de tipo trnasversal, se anida en el proyecto de investigación: "Factores clínicos-epidemiológicos asociados con la severidad (sic.) y la presentación de la leptospirosis en los municipios del Urabá antioqueño, 2009-2012", que en adelante se denominará proyecto macro, el cual lo ejecuta el Instituto Colombiano de Medicina Tropical en los municipios del eje bananero desde el año 2010. Los datos aquí presentados corresponden a los resultados del estudio socioepidemiológico y de factores de riesgo de los casos de leptospirosis diagnosticados por el proyecto macro procedentes del municipio de Turbo. El estudio fue llevado a cabo en febrero y marzo del 2012.

Los casos del proyecto macro se confirmaron por: serología positiva para una o más serovariedades incluidas en el panel de evaluación por la prueba de microaglutinación (MAT) (3), considerándose positiva el alza cuádruple de títulos en muestra convaleciente y un título igual o superior a 1:400 en el caso de disponerse de una sola muestra. En el panel de evaluación serológica por MAT se incluyeron 11 serovariedades representativas de cinco especies patógenas de Leptospira: L. interrogans Copenhageni, Canicola, Hardjo, Pomona y Bratislava; L. santarosai Shermani; L. noguchii Panama; L. kirschneri Cynopteri y Grippotyphosa; L. borgpetersenii Ballum y Tarassovi. Se consideraron como puntos de corte de resultado positivo: a) un título de 1:100 obtenido con, al menos, una de las serovariedades representativas de las especies probadas; b) prueba serológica positiva de inmunofluorescencia indirecta (IFI) para IgM (9), o seroconversión registrada por IFI en fase de convalecencia, y c) cultivo positivo de sangre sembrada en medio semisólido de Fletcher.

De los 116 pacientes procedentes de Turbo que habían sido diagnosticados con leptospirosis dentro del proyecto macro durante los años 2010 y 2011, fue posible ubicar en sus respectivas viviendas a $68(59,5 \%)$. A cada paciente del estudio se le hizo una visita domiciliaria y se le aplicó una encuesta por parte de personal técnico, previamente capacitado. La visita permitió observar las condiciones de la vivienda y referenciar geográficamente su ubicación en el municipio con respecto a la cercanía a fuentes 


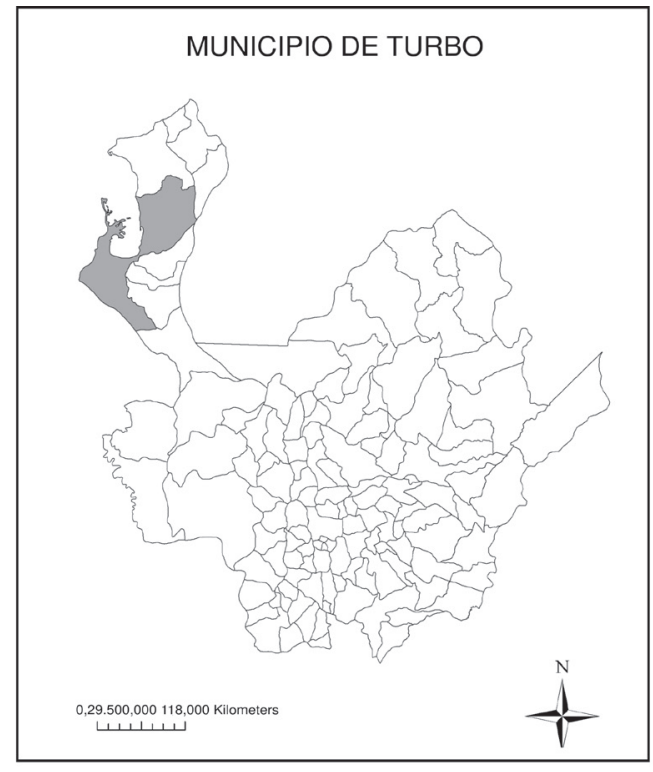

a

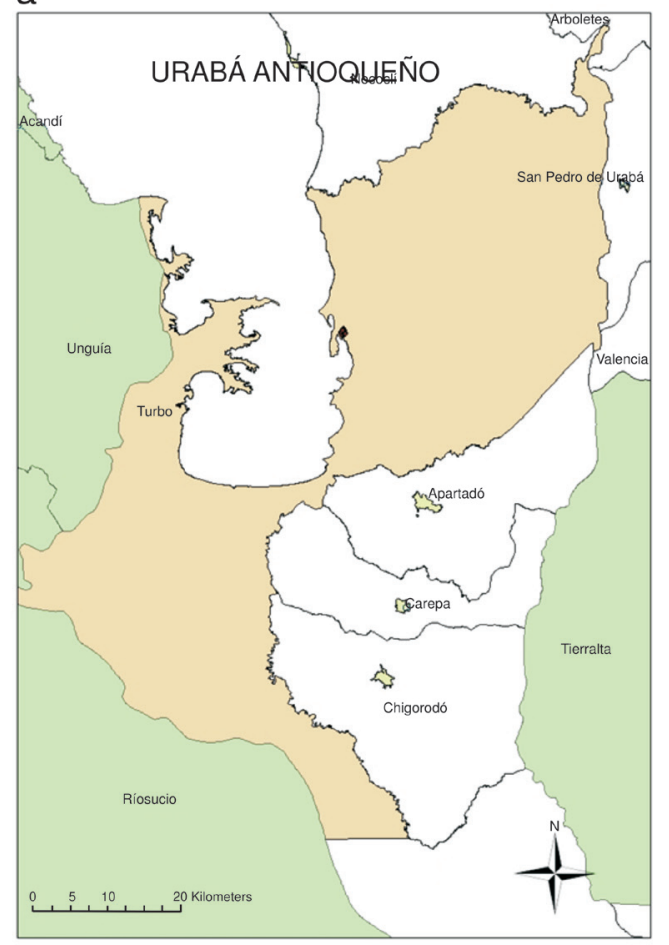

de aguas y explotaciones pecuarias. La encuesta incluyó variables socio-demográficas, hábitos higiénicos, condiciones físicas y de saneamiento básico, convivencia cercana con huéspedes domésticos y animales silvestres, así como presencia y control de roedores sinantrópicos.

\section{Análisis de los datos}

El análisis tuvo un enfoque cuantitativo de tipo exploratorio y se hizo con el programa Stata $11.0^{\circledR}$.
Figura 1. a. Ubicación geográfica de los municipios de Antioquia y de la subregión del Urabá antioqueño, donde se resalta el municipio de Turbo. b. Detalle de la ubicación del municipio de Turbo.

b

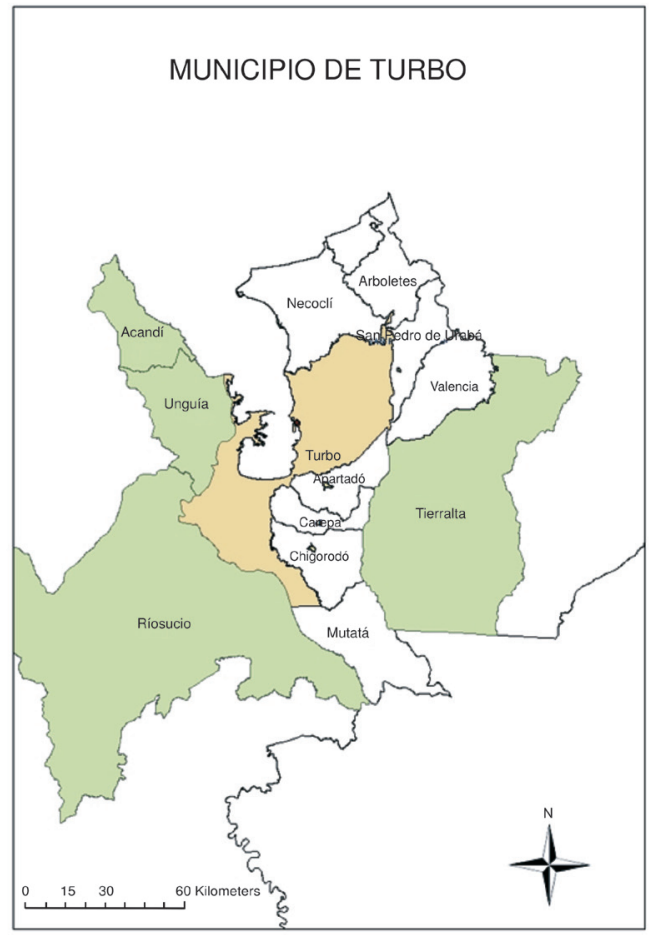

Para efectos del análisis en el presente estudio, los 68 pacientes entrevistados se distribuyeron en dos grupos: uno con 48 casos de manejo hospitalario $(70,6 \%)$ y otro con 20 casos de manejo ambulatorio (29,4\%).

Para elanálisis univariado de datos, se establecieron frecuencias y porcentajes; para el análisis bivariado se practicó la prueba de ji al cuadrado con su respectivo valor de $p$, así como la razón de momios 


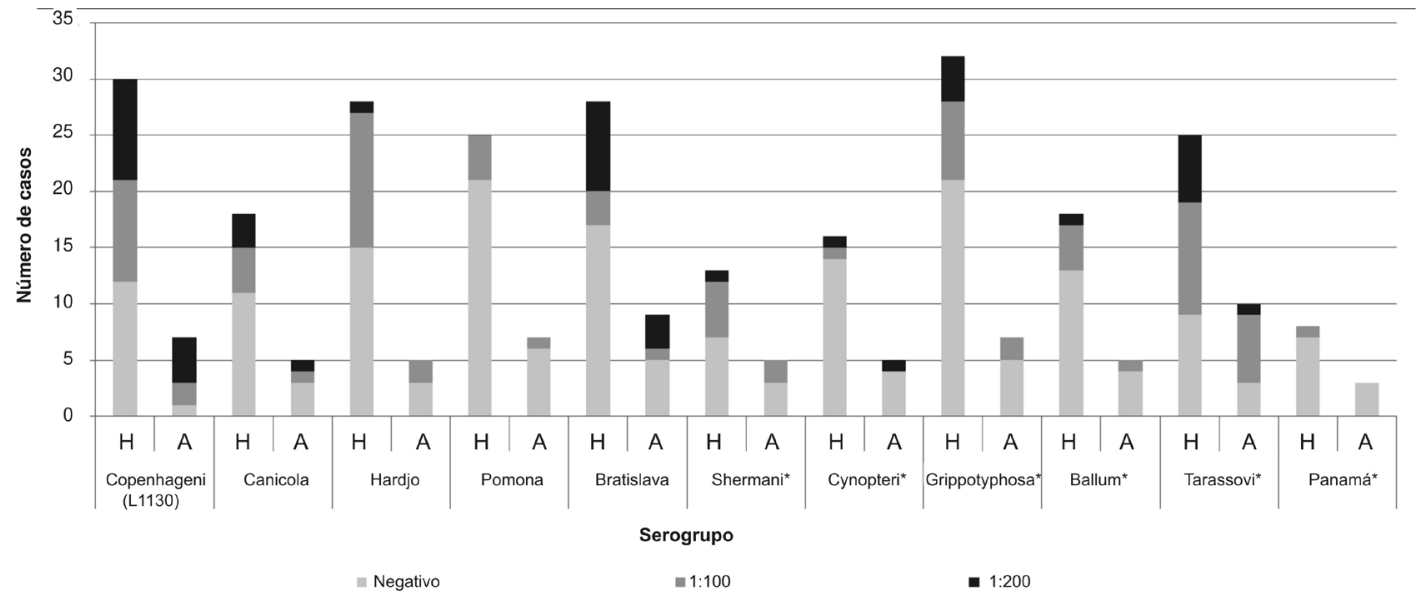

Figura 2. Títulos de anticuerpos para 11 serogrupos de Leptospira spp. en dos series de casos de leptospirosis de manejo ambulatorio y de manejo hospitalario. Los serogrupos de origen silvestre se señalan con asterisco. Turbo, Colombia, 2010-2011.

(odds ratios, OR) con su intervalo de confianza del $95 \%$, para establecer asociación entre la condición de manejo del paciente (hospitalizado o ambulatorio) y las variables independientes.

Se hizo un análisis multivariado exploratorio con el fin de identificar aquellas variables que pudieran explicar la condición de requerir hospitalización en los casos de leptospirosis. Para ello se hizo una regresión logística usando el método de selección hacia adelante (Forward Selection, Wald). Se tomaron como referencia aquellas variables independientes que en el análisis bivariado presentaron un valor de p menor de 0,25 (criterio de Hosmer-Lemeshow, 1989) o que, a criterio de los investigadores, pudieran explicar esta condición.

\section{Consideraciones éticas}

Para la aplicación de la encuesta se contó con la aprobación de los encuestados (persona adulta responsable), quienes firmaron el formulario como señal de aprobación, haciendo especial énfasis en el derecho a la información, la privacidad de la familia y la negativa a participar en el estudio. Este protocolo fue revisado y aprobado por el Comité de Ética del Instituto Colombiano de Medicina Tropical, Universidad CES.

\section{Resultados}

De los 116 casos de leptospirosis registrados en las instituciones de salud del municipio de Turbo entre 2010 y 2011, fue posible encuestar el 59,5\% (68/116). Los 48 casos restantes se excluyeron por cambio de número telefónico o de residencia. Aunque se pretendió que la encuesta fuera respondida preferencialmente en forma directa por quien sufrió leptospirosis, en 17 casos no fue posible hacerlo, porque cinco de ellos habían fallecido al momento de la encuesta (tres durante la hospitalización como consecuencia de las complicaciones asociadas con leptospirosis) y otros 12 casos eran menores de edad. En estos 17 casos, un familiar o acudiente atendió al encuestador.

Los casos eran en su mayoría adultos (edad media de 31,3 años, con desviación estándar de 16,9, oscilando entre 5 y 82 años) y hombres (67,6\%: 46/68). El 70,6 \% (48/68) de los casos requirieron hospitalización y presentaron una mayor frecuencia de manifestaciones clínicas relacionadas con el síndrome de Weil $(21,7 \%)$, compromiso hepático $(13,4 \%)$, compromiso renal $(8,7 \%)$ y compromiso respiratorio $(8,7 \%)$. La letalidad fue de $4,4 \%$ (3/68).

Con respecto a la evaluación serológica realizada por MAT con cinco especies representadas en el panel diagnóstico, se encontró serología positiva absoluta para todas las especies incluidas en la evaluación. L. interrogans Copenhageni obtuvo el $28,6 \%$ de serologías positivas; Canicola, el 11,1\%; Hardjo, el 20,6 \%; Pomona, el 6,3\%, y Bratislava, el $17,5 \%$; $L$. santarosai Shermani, el 9,5\%; $L$. kirschneri Cynopteri, el 3,2 \%, y Grippotyphosa, el 17,5\%; por su parte, L. borgpetersenii Ballum obtuvo el 7,9 \%; Tarassovi, el 25,4 \%, y L. noguchii Panama, el 1,6\%. En la figura 2 se muestra la distribución de estas frecuencias por grupos, evidenciándose que los niveles de resultados positivos fueron mayores en los casos de leptospirosis que requirieron hospitalización.

El $67,6 \%$ de la población de encuestados (51 casos y 17 familiares-convivientes) pertenecían al grupo étnico afrocolombiano, 25,0 \% a población 
mestiza, 2,9 \% eran indígenas y el 4,4 \% no autodefinió su carácter étnico. La edad promedio de los encuestados fue de 31,3 años y el 67,6\% eran hombres. El 98,5\% tenía un año o más de residencia en la zona de Urabá. Sobre la condición de hacinamiento evidenciada por el número de personas convivientes, se determinó que 55,4 $\%$ compartía con más de cinco personas una vivienda de máximo dos cuartos. En cuanto al tipo de afiliación al sistema de seguridad social en salud, se encontró que el 53,6 \% estaba vinculado al régimen subsidiado. La distribución por estrato socioeconómico fue principalmente para el estrato bajo $(85,2 \%)$ y el $17,7 \%$ de los encuestados se dedicaba a labores agrícolas. No se encontró diferencia estadísticamente significativa entre el grupo de casos hospitalizados y el grupo de manejo ambulatorio para las variables socioeconómicas que se analizaron (cuadro 1).

En cuanto a las actividades identificadas como hábitos de riesgo, se encontró que el 59,1\% estuvo expuesto a colecciones de agua como depósitos de agua estancada (15,9\%) y río o quebrada $(29,5$ $\%)$. El 25,8 \% manifestó haber tenido abrasiones en la piel en los últimos dos meses y en el 52,3 $\%$ su trabajo los obligaba a caminar descalzos, pero también una proporción similar $(50,7 \%)$ por costumbre caminaban descalzos dentro y fuera de la casa. El 61,2\% de los encuestados se lavaba las manos antes y después de usar el baño; el 50,9\% hervía el agua antes de consumirla, las personas que no lo hacían la consumían directamente del tanque de agua o pozo (73,5 \%), del grifo (16,3\%) y de otras fuentes como río, quebrada, aljibe u otros recipientes de almacenamiento (10,2\%). El $86,6 \%$ se lavaba las manos antes de preparar los alimentos y el $86,4 \%$ lavaba los recipientes antes de usarlos. No hubo asociación estadísticamente significativa entre estos hábitos de riesgo y la condición de manejo ambulatorio u hospitalario de los casos de leptospirosis.

La costumbre de consumir alimentos del día anterior, independientemente del lugar donde permanecieron en la noche, la practicaba el 48,4\% de los encuestados; los hospitalizados (60,9\%) refirieron esta costumbre con mayor frecuencia, sin ser esta diferencia estadísticamente significativa al compararla con los casos ambulatorios.

El $71 \%$ de las viviendas donde habitaban los casos se ubicaban en la zona urbana. En la figura 3 se muestra la geodistribución de las viviendas de los pacientes en el municipio, evidenciándose una mayor proporción en esta zona. Sobre la localización de las viviendas en zonas de alto riesgo de inundación, se encontró que 74,2 \% estaban al lado de un río o del mar. Asimismo, se

Cuadro 1. Distribución de las condiciones socio-demográficas según casos de leptospirosis hospitalizados y ambulatorios. Turbo, Colombia, 2010-2011.

\begin{tabular}{|c|c|c|c|c|c|c|}
\hline \multirow{2}{*}{$\begin{array}{l}\text { Variable } \\
\text { hospitalizados }\end{array}$} & \multicolumn{2}{|c|}{ Casos hospitalizados } & \multicolumn{2}{|c|}{ Casos ambulatorios } & \multirow{2}{*}{$\begin{array}{c}\chi^{2} \text { o prueba } \\
\text { exacta de Fisher }\end{array}$} & \multirow{2}{*}{$\begin{array}{l}\text { Casos } \\
\text { Prueba } p\end{array}$} \\
\hline & $\mathbf{n}$ & $\%$ & $\mathbf{n}$ & $\%$ & & \\
\hline \multicolumn{7}{|c|}{ Miembros por familia } \\
\hline 1 a 2 & 8 & 17 & 4 & 22,2 & 1,22 & 0,607 \\
\hline 3 a 4 & 14 & 29,8 & 3 & 16,7 & & \\
\hline 5 o más & 25 & 53,2 & 11 & 61,1 & & \\
\hline \multicolumn{7}{|c|}{ Tipo de afiliación al SGSSS } \\
\hline Contributivo & 17 & 39,5 & 9 & 69,2 & 2,62 & 0,060 \\
\hline Subsidiado & 26 & 60,5 & 4 & 30,8 & & \\
\hline \multicolumn{7}{|c|}{ Estrato socioeconómico de la vivienda } \\
\hline Bajo & 41 & 87,2 & 11 & 78,6 & 1,66 & 0,518 \\
\hline Medio & 5 & 10,6 & 3 & 21,4 & & \\
\hline Alto & 1 & 2,1 & 0 & 0 & & \\
\hline \multicolumn{7}{|l|}{ Ocupación } \\
\hline Labores agrícolas & 10 & 22,7 & 1 & 5,6 & 8,71 & 0,222 \\
\hline Oficios varios & 10 & 22,7 & 2 & 11,1 & & \\
\hline Estudiante & 8 & 18,2 & 4 & 22,2 & & \\
\hline Enfermero & 2 & 4,5 & 1 & 5,6 & & \\
\hline Ama de casa & 2 & 4,5 & 2 & 11,1 & & \\
\hline Independiente & 1 & 2,3 & 1 & 5,6 & & \\
\hline Guía canino & 1 & 2,3 & 3 & 16,7 & & \\
\hline Otro & 10 & 22,7 & 4 & 22,2 & & \\
\hline
\end{tabular}

SGSSS: Sistema General de Seguridad Social en Salud 


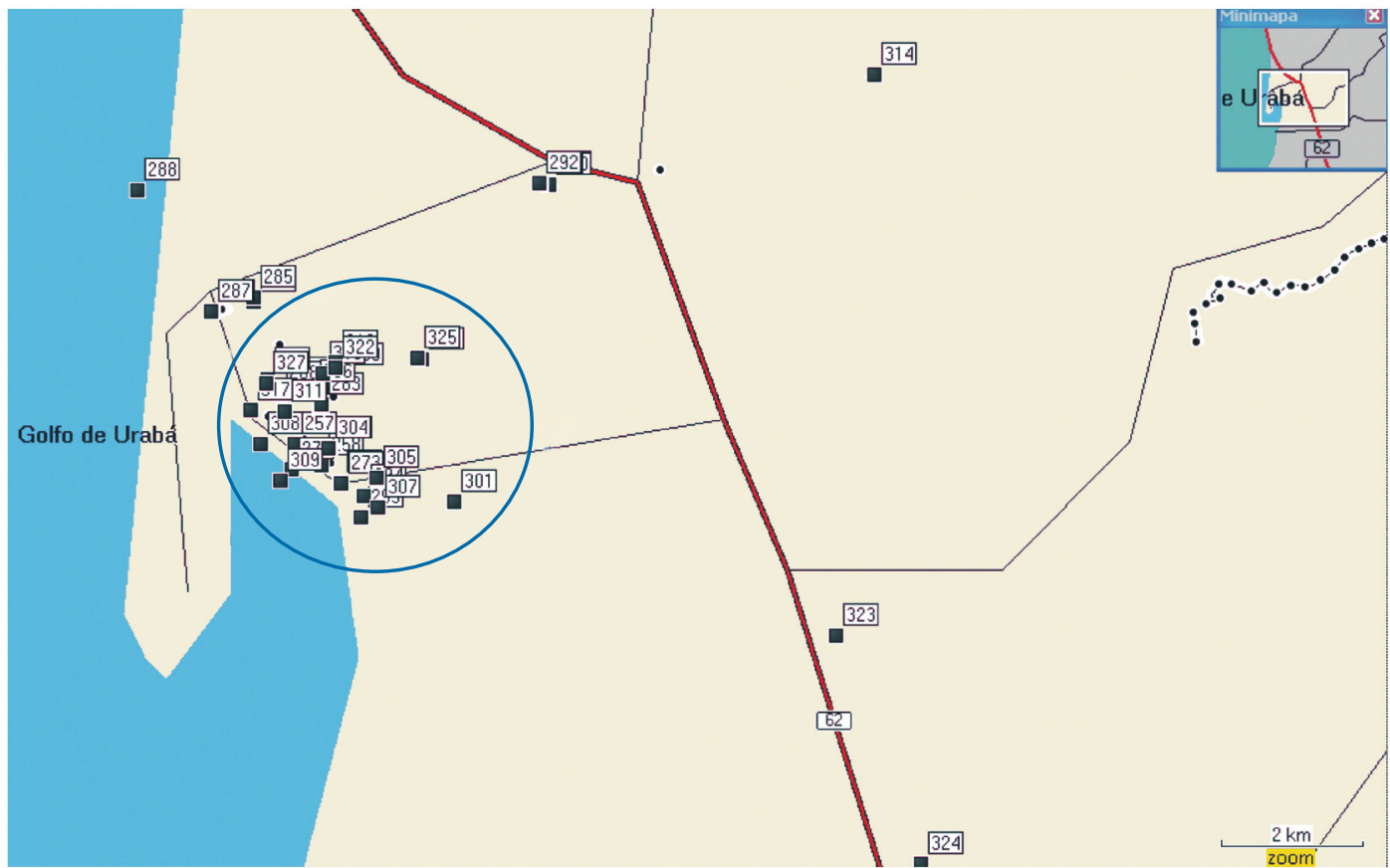

Figura 3. Geodistribución de las viviendas (en cuadro negro) de los casos positivos para leptospirosis. La línea gruesa corresponde a la vía principal al mar y, las líneas delgadas, a las vías alternas. Se delimita en el círculo la zona urbana y periurbana del municipio en donde se localiza la mayoría de las viviendas de los encuestados. Turbo, Colombia, 2010-2011.

indagó sobre inundaciones, encontrándose que $64,5 \%$ de las viviendas se inundaron en los últimos seis meses. Se observó que el $74,6 \%$ de las viviendas estaban rodeadas de aguas estancadas y sus habitantes refirieron que las entidades de saneamiento del municipio no habían hecho ningún tipo de intervención en estas aguas en los últimos dos años. En los alrededores inmediatos del 65,2 $\%$ de las viviendas, existen producciones agropecuarias. El 97,7 \% de estos establecimientos agropecuarios se encuentra a 500 metros o menos de distancia de las viviendas de los casos.

Los materiales más frecuentemente usados para la construcción de las paredes de las viviendas fueron adobe-cemento $(64,4 \%)$, pisos en cerámica $(55,2$ $\%)$ y techo con tejas de asbesto-cemento $(64,6 \%)$; sin embargo, el 92,5\% de las viviendas no tenía capacidad para evitar el acceso de animales al interior, evidenciándose deficiencias de soporte, contención y cerramiento completo de puertas y ventanas.

En cuanto al saneamiento básico, el 56,7 \% de las viviendas no tenía suministro de agua potable con conexión por acueducto y el $75,7 \%$ de este grupo se proveía de aguas lluvias. El 59,4 \% de las viviendas tenía pozo o tanque para recolección de aguas tanto de acueducto como de lluvias y, de estos, la mitad $(50,0 \%)$ permanecía cubierto con tapa o protección. Se encontró que el 82,4 \% de los casos ambulatorios consumía agua extraída directamente del tanque o pozo; asimismo, el $51,5 \%$ de los casos hospitalizados manifestó tener pozo, aunque se encontró una diferencia entre tener pozo y ser manejado ambulatoriamente $(\mathrm{OR}<1)$ presentándose como un factor protector para leptospirosis de manejo hospitalario (cuadro 2). El $65,7 \%$ de las viviendas no contaba con conexión a alcantarillado y, de estas, el 52,4\% tenía letrina. El $77,6 \%$ de las viviendas tenía servicio de recolección de basuras dos veces por semana y el $22,4 \%$ restante no lo tenía; de estas, el 60,0 \% quemaba la basura, el 20,0 \% la arrojaba al río y el otro $20,0 \%$ le daba otros manejos, como depositarlas a campo abierto o arrojarlas al mar.

Frente a la presencia o ausencia de roedores, el $85,3 \%$ de los encuestados manifestó haber visto roedores dentro de su vivienda; de estos, el 91,8 $\%$ realizaba control químico, mecánico o ambos. Al momento de hacer el control de roedores, 76,3\% refirió manipular los animales muertos con guantes, bolsa o pala. No hubo diferencia estadísticamente significativa entre la presencia de roedores en la vivienda y la condición de hospitalización entre los casos de leptospirosis $(p=0,240)$. La variable 
Cuadro 2. Distribución de las variables de condiciones de viviendas según casos de leptospirosis hospitalizados y ambulatorios. Turbo, Colombia, 2010-2011.

\begin{tabular}{|c|c|c|c|c|c|c|}
\hline \multirow[t]{2}{*}{ Variable } & \multicolumn{2}{|c|}{ Casos hospitalizados } & \multicolumn{2}{|c|}{ Casos ambulatorios } & \multirow[t]{2}{*}{ OR $\left(\mathrm{IC}_{95 \%}\right)$} & \multirow[t]{2}{*}{ Prueba $\mathrm{p}$} \\
\hline & $\mathbf{n}$ & $\%$ & $\mathbf{n}$ & $\%$ & & \\
\hline \multicolumn{7}{|c|}{$\begin{array}{l}\text { Las puertas y ventanas de la vivienda } \\
\text { permiten el ingreso de animales }\end{array}$} \\
\hline Sí & 45 & 93,8 & 17 & 89,5 & 1,76 & 0,617 \\
\hline No & 3 & 6,3 & 2 & 10,5 & $(0,27-11,5)$ & \\
\hline \multicolumn{7}{|c|}{ Tiene pozo de agua en su casa } \\
\hline Sí & 24 & 51,1 & 14 & 82,4 & 0,22 & $0,022^{*}$ \\
\hline No & 23 & 48,9 & 3 & 17,6 & $(0.06-17,6)$ & \\
\hline \multicolumn{7}{|c|}{ El pozo permanece tapado } \\
\hline Sí & 10 & 41,7 & 9 & 64,3 & 2,52 & 0,179 \\
\hline No & 14 & 58,3 & 5 & 35,7 & $(0,65-9,83)$ & \\
\hline \multicolumn{7}{|c|}{ Conexión a alcantarillado } \\
\hline Sí & 14 & 29,2 & 9 & 47,4 & 2,19 & 0,157 \\
\hline No & 34 & 70,8 & 10 & 52,6 & $(0,73-6,53)$ & \\
\hline \multicolumn{7}{|c|}{$\begin{array}{l}\text { Existen producciones agropecuarias } \\
\text { en los alrededores de la vivienda }\end{array}$} \\
\hline Sí & 33 & 70,2 & 10 & 52,6 & 2,12 & 0,175 \\
\hline No & 14 & 29,8 & 9 & 47,4 & $(0,71-6,35)$ & \\
\hline
\end{tabular}

* Valor de p menor de 0,05

que exploraba el tipo de control para roedores (químico,mecánico o ninguno), tampoco presentó diferencias estadísticamente significativas entre ambos grupos $(p=0,313)$.

Con respecto a la tenencia de animales domésticos, el $68,7 \%$ de los dueños de las viviendas manifestó tener animales como perros, gatos y aves de corral. En este mismo sentido, el 40,9\% de los animales permanecía y dormía dentro de la vivienda, y de ellos, el 21,7 \% dormía en las habitaciones. Con respecto a la presencia de animales no domésticos, se encontró que el $59,7 \%$ de las viviendas albergaba especies de aves silvestres, pequeños primates y ratones de monte, entre otros. El análisis por grupos registró que el 66,7 \% de los casos hospitalizados residía en viviendas que poseían este tipo de animales, pero no hubo diferencia estadísticamente significativa entre el haber sido hospitalizado o haber sido manejado ambulatoriamente por leptospirosis $y$ tener animales no domésticos en la vivienda $(p=0,065)$. No obstante, esta última condición representó $2,75(0,92-8,18)$ veces el riesgo de presentar leptospirosis de manejo hospitalario. En el cuadro 3 se muestra la distribución de casos hospitalizados y ambulatorios con respecto a las condiciones evaluadas del entorno ambiental de las viviendas, sus frecuencias absolutas, proporciones, OR con su respectivo IC y el valor de $\mathrm{p}$.

El 59,6\% de los casos hospitalizados desarrollaba actividades que implicaban caminar descalzo, y esta condición representó 2,95 $(0,94-9,22)$ veces el riesgo para leptospirosis de manejo hospitalario, sin asociación estadísticamente significativa $(p=0,058)$. Ahora bien, el 60,4\% de los casos hospitalizados acostumbraba caminar descalzo dentro y fuera de la vivienda, mientras que el $26,3 \%$ de los casos ambulatorios lo hacía. Esta condición de caminar descalzo dentro y fuera de la vivienda representó $4,27(1,32-13,82)$ veces el riesgo de tener leptospirosis de manejo hospitalario, siendo esta diferencia estadísticamente significativa $(p=0,012)$ (cuadro 4).

Finalmente, las variables presentadas en los cuadros 2, 3 y 4 se incluyeron en un modelo de regresión logística (análisis multivariado), aun cuando el análisis bivariado no hubiera arrojado diferencia estadísticamentesignificativa. Esteanálisisevidenció que la variable tener animales no domésticos dentro de la vivienda representa 4,22 $(1,13-15,72)$ veces el riesgo de requerir hospitalización, comparado con los casos ambulatorios que manifestaron no tener este tipo de animales $(p=0,032)$.

\section{Discusión}

Este estudio de carácter exploratorio de factores de riesgo para los pacientes con leptospirosis que requirieron manejo hospitalario en el municipio de Turbo, pone de manifiesto que, para esta población, el hábito de caminar descalzo y la presencia de fauna silvestre en las viviendas, pueden estar relacionados con casos de leptospirosis de curso moderado a grave. Además, explora relaciones 
Cuadro 3. Distribución de variables del entorno de las viviendas según casos de leptospirosis hospitalizados y ambulatorios. Turbo, Colombia, 2010-2011.

\begin{tabular}{|c|c|c|c|c|c|c|}
\hline \multirow[t]{2}{*}{ Variable } & \multicolumn{2}{|c|}{ Casos hospitalizados } & \multicolumn{2}{|c|}{ Casos ambulatorios } & \multirow[t]{2}{*}{ OR $\left(\mathrm{IC}_{95 \%}\right)$} & \multirow[t]{2}{*}{ Prueba p } \\
\hline & & $\%$ & & $\%$ & & \\
\hline \multicolumn{7}{|c|}{$\begin{array}{l}\text { Presencia de animales no } \\
\text { domésticos en la vivienda }\end{array}$} \\
\hline Sí & 32 & 66,7 & 8 & 42,1 & 2,75 & 0,065 \\
\hline No & 16 & 33,3 & 11 & 57,9 & $(0,92-8,18)$ & \\
\hline \multicolumn{7}{|c|}{$\begin{array}{l}\text { Existencia de ríos y quebradas } \\
\text { cerca de la vivienda }\end{array}$} \\
\hline Sí & 38 & 79,2 & 11 & 61,1 & 2,42 & 0,205 \\
\hline No & 10 & 20,8 & 7 & 38,9 & $(0,75-7,84)$ & \\
\hline \multicolumn{7}{|c|}{$\begin{array}{l}\text { Aguas estancadas en los } \\
\text { alrededores de la vivienda }\end{array}$} \\
\hline Sí & 38 & 79,2 & 12 & 63,2 & 2,22 & 0,217 \\
\hline No & 10 & 20,8 & 7 & 36,8 & $(0,69-7,1)$ & \\
\hline
\end{tabular}

Cuadro 4. Distribución de variables de hábitos de riesgo según casos de leptospirosis hospitalizados y ambulatorios. Turbo, Colombia, 2010-2011.

\begin{tabular}{|c|c|c|c|c|c|c|}
\hline \multirow[t]{2}{*}{ Variable } & \multicolumn{2}{|c|}{ Casos hospitalizados } & \multicolumn{2}{|c|}{ Casos ambulatorios } & \multirow[t]{2}{*}{ OR $\left(\right.$ IC $\left._{95 \%}\right)$} & \multirow[t]{2}{*}{ Prueba $p$} \\
\hline & & $\%$ & $\mathbf{n}$ & $\%$ & & \\
\hline \multicolumn{7}{|c|}{$\begin{array}{l}\text { Acostumbra a caminar descalzo } \\
\text { o su trabajo lo obliga a hacerlo }\end{array}$} \\
\hline Sí & 28 & 59,6 & 6 & 33,3 & 2,95 & 0,058 \\
\hline No & 19 & 40,4 & 12 & 66,7 & $(0,94-9,22)$ & \\
\hline \multicolumn{7}{|c|}{$\begin{array}{l}\text { Camina descalzo dentro y fuera } \\
\text { de la casa }\end{array}$} \\
\hline Sí & 29 & 60,4 & 5 & 26,3 & 4,27 & $0,012^{*}$ \\
\hline No & 19 & 39,6 & 14 & 73,7 & $(1,32-13,82)$ & \\
\hline \multicolumn{7}{|c|}{$\begin{array}{l}\text { Exposición a depósitos de agua } \\
\text { con fines ocupacionales o recreativos }\end{array}$} \\
\hline Sí & 31 & 64,6 & 8 & 44,4 & 2,28 & 0,205 \\
\hline No & 17 & 35,4 & 10 & 55,6 & $(0,76-6,87)$ & \\
\hline
\end{tabular}

* valor de p menor de 0,05

con aspectos que pueden ser determinantes de la exposición a Leptospira spp. en esta zona endémica colombiana, tales como aspectos sociodemográficos, hábitos, condiciones físicas y de saneamiento de la vivienda, hacinamiento, fuentes de agua potable, presencia y control de roedores sinantrópicos, y convivencia cercana con huéspedes domésticos y silvestres, todos ellos factores que son ampliamente reconocidos como condicionantes de la presentación de la leptospirosis a nivel mundial $(1,5,10,11)$.

Los resultados desprendidos de este trabajo, sobre la presentación de leptospirosis en las personas que exhiben factores determinantes sociales y ambientales clásicamente reconocidos como condicionantes de su riesgo para adquirirla, reafirman el conocimiento generado de investigaciones similares, realizadas en contextos latinoamericanos, que concluyen que vivir en zonas de crecimiento subnormal con condiciones ambientales deficientes favorece la proliferación de poblaciones de roedores, los cuales son los principales reservorios de la bacteria $(5,12)$. Del mismo modo que en otros contextos latinoamericanos, al presentar zonas con urbanización deficiente y falta de saneamiento ambiental, el municipio de Turbo posee todas las condiciones para que los roedores actúen como diseminadores de la enfermedad entre la población humana, hecho corroborado por la presencia de anticuerpos para $L$. interrogans Copenhageni (principalmente asociado a roedores) en el 28,6 \% de los casos registrados en este estudio.

Las condiciones físicas de las viviendas del municipio, además de ser un factor relacionado con los niveles de pobreza, también permitieron conocer el riesgo al que están expuestas las 
personas que en ellas viven. Este estudio evidenció que el $92,5 \%$ de las viviendas donde residían los casos, permitían el fácil acceso de animales a su interior, lo cual a su vez se traduce probablemente en riesgo de contraer diferentes tipos de bacterias diseminadas por animales silvestres y domésticos. Dichos hallazgos son similares a lo informado por otros autores, en cuanto a que la infraestructura urbana inadecuada es un factor de riesgo asociado a la infección por Leptospira spp. $(5,12)$.

Por otro lado, también en el estudio llevado a cabo en otro municipio de la zona se encontró asociación de serología positiva para Leptospira spp. y habitar viviendas que tenían almacenamiento de cosechas sin cerramiento completo (8). Esto puede deberse a que los roedores ingresan a las viviendas por aberturas en paredes y techos atraídos por cosechas o alimentos que pueden estar guardados sin cubrimiento o dentro de embalajes expuestos a las roeduras, contaminándolos así con su orina y contaminando a su vez las sendas por donde transitan en su búsqueda de alimento. Además, en el presente estudio, este factor de riesgo se relaciona con la mayor frecuencia encontrada entre los que comen alimentos preparados el día anterior, independientemente de donde hubiesen sido almacenados, y la presentación de leptospirosis de manejo hospitalario.

Aunque otras variables consideradas en el análisis bivariado y multivariado, como existencia de ríos y quebradas en los alrededores de las viviendas, haber sufrido inundación en los seis meses previos a la presentación de leptospirosis y presencia de aguas estancadas sin tratamiento en los alrededores de la vivienda, no presentaron asociación estadísticamente significativa con haber presentado leptospirosis de manejo hospitalario, sí hubo una frecuencia importante de estas que dan cuenta de la condición de vulnerabilidad de la población objeto de este estudio. Además, estos son factores que deben considerarse como factores condicionantes para la alta rotación de población que se presenta en la zona; es decir, las familias que construyen sus viviendas en asentamientos subnormales deben migrar de un lugar a otro, en búsqueda de sitios más seguros, lo que influye también en la poca durabilidad de los materiales usados para la construcción de las viviendas y la poca o nula seguridad que estas ofrecen para impedir el libre acceso de animales al interior de los espacios domésticos.

En Perú (11) y en la Costa Atlántica colombiana (13), se ha establecido que el contacto con aguas superficiales o de inundación, representa un riesgo epidemiológico para leptospirosis, permitiendo concluir que la presencia de aguas estancadas - la circulación de ríos o quebradas cerca a la vivienda, es un factor de riesgo para que las personas puedan contraer leptospirosis. En el presente estudio la frecuencia de este antecedente fue similar para los casos de manejo ambulatorio y hospitalario. Aunque los exámenes moleculares de aguas residuales y estancadas, llevados a cabo anteriormente en la zona por nuestro grupo, han evidenciado circulación de Leptospira spp. en la región del Urabá (14), es necesario realizar investigaciones posteriores para vigilar fuentes ambientales que identifiquen la circulación de Leptospira spp. en el mismo momento y lugar de presentación de casos de leptospirosis, para así relacionar estas dos condiciones, tal como fue registrado en zona urbana de lquitos en Perú (11). Este conocimiento es básico para implementar acciones de prevención y control inmediato del riesgo, por parte de las autoridades ambientales del municipio.

Hubo una mayor frecuencia de casos hospitalarios que no tenían servicio de alcantarillado, por lo cual la disposición de los excrementos se hacía a campo abierto y en aguas estancadas en los alrededores de la vivienda. En forma similar, en Brasil durante una epidemia urbana (15), se relacionó el riesgo para contraer leptospirosis con las alcantarillas abiertas, el pobre sistema de drenaje de aguas lluvias asociado con inundaciones, la falta de servicios de recolección de basuras, y la presencia de ratas en los hogares. Al menos una de estas fuentes ambientales de contaminación se encontró en todas las viviendas encuestadas en el municipio de Turbo, dando cuenta de la marginalidad de estos asentamientos, lo que puede haber contribuido a la concentración del riesgo de infección de los casos de leptospirosis estudiados y evidencia la exposición potencial a Leptospira spp. de toda la población del estudio que presentó leptospirosis. Aunque hubo una relación de aparente protección de los casos de manejo ambulatorio con la presencia de pozo de agua en su vivienda, pudiendo estar condicionado al que este permaneciera tapado, es difícil concluir que la disponibilidad de este recurso dentro de la vivienda se comporte como protector, porque muchos de los pozos son artesanales sin un adecuado mantenimiento.

Por su parte, la leptospirosis está catalogada como enfermedad de riesgo ocupacional; las actividades agrícolas, de albañilería y otras relacionadas con 
el contacto con suelos y aguas, son las de mayor riesgo de exposición a Leptospira spp. (1, 2), lo cual concuerda con la situación encontrada en el presente estudio, en el cual la ocupación agropecuaria predominó en el grupo de casos de leptospirosis de manejo hospitalario, sin encontrar diferencias entre ambos grupos.

El hábito de caminar descalzo está muy arraigado en comunidades afrodescendientes e indígenas, pero caminar sin protección es un riesgo ampliamente reconocido para exponerse a Leptospira spp. (1), por favorecer una vía de ingreso para una mayor carga bacteriana. En este estudio no sólo se evidencia como riesgo para la presentación de los casos de leptospirosis, sino también, se relacionó significativamente con requerir manejo hospitalario de la leptospirosis. Debe tenerse en cuenta que, en el caso de las personas dedicadas a labores agrícolas, este hábito puede presentarse también en ambientes donde estén presentes fauna silvestre o fuentes de agua contaminadas con orina de animales de explotación pecuaria, por lo que el riesgo en este grupo puede ser de tipo ocupacional y no necesariamente domiciliario, como se evidenció en este estudio. Este aspecto debe tenerse en cuenta para futuras investigaciones.

El hábito de caminar descalzo también puede ser potenciado por la tenencia de animales de todo origen, por la presencia de roedores deambulando por la vivienda de pisos de tierra y cerramiento incompleto, por saneamiento deficiente del entorno y por hábitos de riesgo frecuentes en las personas del grupo de casos de leptospirosis del municipio de Turbo. Con respecto a los hábitos de riesgo, se evidenció que en esta población existe un conocimiento parcialmente adecuado sobre las conductas que deben seguirse en relación con hábitos higiénicos y sanitarios, pero no necesariamente el conocimiento de estos hábitos garantiza que efectivamente sean practicados por todos los miembros de la familia. Durante la aplicación de la encuesta no se verificó la práctica efectiva de los hábitos higiénicos expresados por la persona que respondió la encuesta.

No obstante las anteriores evidencias de carácter exploratorio de factores de riesgo para la población de estudio frente a leptospirosis, el principal aporte del presente trabajo es haber obtenido pruebas del papel de la fauna silvestre que, por acción antropogénica, es introducida en ambientes urbanos. Se reconoce que en el caso de las enfermedades zoonóticas como la leptospirosis hay una diversa gama de reservorios, tanto de naturaleza doméstica como pecuaria, los cuales pueden emerger con nuevas dinámicas de presentación clínica cuando son introducidas fuentes de la bacteria de origen silvestre. Esto implica que la epidemiología de esas zoonosis sea más compleja en las zonas en las que se presenta este fenómeno derivado del comportamiento humano. El reconocimiento de este factor de riesgo, que en algunas ocasiones pasa desapercibido para la mayoría de los investigadores en salud pública, es un aporte del presente estudio para recomendar que se lleven a cabo acciones de prevención y control por parte de las autoridades ambientales para detener esta práctica.

Las acciones que se emprendan deben estar acompañadas, además, de una vigilancia activa de la fauna silvestre, implementando en lo posible herramientas moleculares de diagnóstico disponibles en el medio. Además, es recomendable establecer relaciones interdisciplinarias y transdisciplinarias que permitan ampliar nuestro conocimiento sobre posibilidades de diseminación de esta zoonosis potenciada por introducción de nuevos aislamientos de virulencia desconocida que entran en contacto con reservorios y huéspedes expuestos a Leptospira spp., entre los que se cuenta el hombre. Esto debe estar acompañado de campañas educativas, sostenibles en el tiempo, dirigidas a las comunidades de áreas rurales y urbanas periféricas. Este aspecto debe tenerse en cuenta debido a que ancestral y culturalmente las comunidades afrocolombianas han mantenido la convivencia cercana con los animales de origen silvestre. Estas comunidades no consideran que su accionar afecte su salud al no tener la percepción de riesgo de que animales silvestres, introducidos por ellos a los ambientes domésticos, sean fuentes de un sinnúmero de enfermedades zoonóticas, como es el caso particular de la leptospirosis.

Hasta el momento no se registran en la literatura científica estudios sobre fauna silvestre que evidencien presencia de leptospirosis en animales provenientes de la zona selvática de la región de Urabá. Hace falta información que presente la importancia de la fauna no doméstica en la cadena de transmisión de la leptospirosis. La detección en los sueros humanos de respuesta de anticuerpos para especies de Leptospira de fuentes no exclusivamente doméstica o pecuaria, tales como 
L. kirschneri, L. borgpetersenii y L. noguchii, demuestra que esta población de casos ha estado o está en contacto con fuentes infecciosas de origen silvestre. Como este hallazgo puede ser diferente en otras áreas geográficas y depende directamente de la frecuencia con que Leptospira spp. esté en la población reservoria de origen selvático, es preciso establecer la circulación de esta en poblaciones de mamíferos como marsupiales y quirópteros, ampliamente distribuidas y abundantes en la zona. En este sentido, el papel de Didelphis marsupialis y de murciélagos frugívoros y hematófagos en la transmisión de leptospirosis en Perú y Brasil, ha sido documentado $(16,17)$ y, por lo tanto, debe ser blanco de atención de investigaciones que se emprendan a futuro.

Si bien se reconocen las limitaciones de este estudio relacionadas con el diseño (estudio descriptivo), disminución de la muestra por pérdida de casos posiblemente debido a los altos índices migratorios de la población de la zona, sesgo de temporalidad (debido a que las encuestas no se hicieron en el momento preciso en que se presentó el síndrome febril) y sesgo de no respuesta del encuestado a todos los ítems del cuestionario (datos perdidos), este es el primer estudio que de modo exploratorio presenta un análisis objetivo de factores de riesgo asociados con la presentación de casos de leptospirosis en el municipio de Turbo y pone en evidencia el papel de la fauna silvestre como factor que puede estar condicionando el espectro de presentaciones complicadas de leptospirosis que están requiriendo hospitalización en el municipio.

Este estudio plantea bases para proponer nuevas investigaciones, diseñar e implementar intervenciones efectivas y específicas para esta zona geográfica que está evidenciando una epidemiología dinámica de contexto particular y complejo que, de igual modo, amerita una intervención en salud pública orientada desde este perfil de riesgos al que se exponen sus habitantes.

\section{Agradecimientos}

Los autores del presente trabajo agradecen al personal auxiliar del Instituto Colombiano Agropecuario, ICA, por su apoyo en el desarrollo de la presente investigación.

\section{Conflicto de intereses}

No existe conflicto de intereses entre los autores, las instituciones financiadoras y el contenido del presente trabajo.

\section{Financiación}

Este proyecto fue financiado por el Instituto Colombiano de Medicina Tropical y por Colciencias (Cod. 325649326207-678).

\section{Referencias}

1. Bharti AR, Nally JE, Ricaldi JN, Matthias MA, Diaz MM, Lovett MA, et al. Leptospirosis: A zoonotic disease of global importance. Lancet Infect Dis. 2003;3:757-71. http://dx.doi. org/10.1016/S1473-3099(03)00830-2

2. Ko Al, Goarant C, Picardeau M. Leptospira: The dawn of the molecular genetics era for an emerging zoonotic pathogen. Nat Rev Microbiol. 2009;7:736-47. http://dx.doi. org/10.1038/nrmicro2208

3. World Health Organization, International Leptospirosis Society. Human leptospirosis: Guidance for diagnosis, surveillance and control 2003. Fecha de consulta: 9 de septiembre de 2012. Disponible en: http://www.med.monash. edu.au/microbiology/staff/adler/leptoguidelines2003.pdf.

4. Bunnell JE, Hice CL, Watts DM, Montrueil V, Tesh RB, Vinetz JM. Detection of pathogenic Leptospira spp infections among mammals captured in the Peruvian Amazon basin region. Am J Trop Med Hyg. 2000;63:255-8.

5. Reis R, Ribeiro G, Felzemburgh R, Santana F, Mohr $\mathbf{S}$, Melández A, et al. Impact of environment and social gradient on Leptospira infection in urban slums. PLoS Negl Trop Dis. 2008;2:e228. http://dx.doi.org/10.1371/journal. pntd.0000228

6. Instituto Nacional de Salud. Sistema de Vigilancia en Salud Pública. Fecha de consulta: 1 de octubre de 2012. Disponible en: http://www.ins.gov.co/lineas-de-accion/ Subdireccion-Vigilancia/sivigila/Paginas/sivigila.aspx.

7. Agudelo-Flórez P, Restrepo-Jaramillo BN, ArboledaNaranjo M. Situación de la leptospirosis en el Urabá Antioqueño colombiano: estudio seroepidemiológico y factores de riesgo en población general urbana. Cad Saúde Pública. 2007;23:2094-102. http://dx.doi.org/10.1590/ S0102-311X2007000900017

8. Padmanabha H, Hidalgo M, Valbuena G, Castañeda E, Galeano A, Puerta $\mathrm{H}$, et al. Geographic variation in risk factors for SFG rickettsial and leptospiral exposure in Colombia. Vector Borne Zoonotic Dis. 2009;9:483-90. http:// dx.doi.org/10.1089/vbz.2008.0092

9. Agudelo-Florez P, Restrepo M, Lotero MA. Evaluación de la prueba de inmunofluorescencia para el diagnóstico de leptospirosis humana. Biomédica. 2006;26:216-23.

10. Johnson MA, Smith H, Joeph P, Gilman RH, Bautista CT, Campos KJ, et al. Environmental exposure and leptospirosis, Peru. Emerg Infect Dis. 2004;10:1016-22. http://dx.doi.org/1010.3201/eid1006.030660

11. Ganoza CA, Matthias MA, Collins-Richards D, Brouwer KC, Cunningham CB, Segura ER, et al. Determining risk for severe leptospirosis by molecular analysis of environmental surface waters for pathogenic Leptospira. PLoS Med. 2006;3:e308. http://dx.doi.org/10.1371/journal. pmed.0030308

12. Maciel EA, de Carvalho AL, Nascimento SF, de Matos RB, Gouveia EL, Reis MG, et al. Household transmission 
of Leptospira infection in urban slum communities. PLoS Negl Trop Dis. 2008;2:e154. http://dx.doi.org/10.1371/ journal.pntd.0000154

13. Epsein PR, Calix O, Blanco J. Climate and disease in Colombia. Lancet. 1995;346:1243-4. http://dx.doi. org/10.1016/S0140-6736(95)91856-6

14. Moreno N, Agudelo Flórez P. Aplicación de las pruebas de PCR convencional simple y múltiple para la identificación de aislamientos de Leptospira spp. en Colombia. Rev Peru Med Exp Salud Pública. 2010;27:548-56.

15. Ko Al, Galvão Reis M, Ribeiro Dourado C, Johnson Jr W, Riley L. Urban epidemic of severe leptospirosis in Brazil.
Lancet. 1999;354:820-5. http://dx.doi.org/10.1016/S01406736(99)80012-9

16. Bunnell JE, Hice CL, Watts DM, Montrueil V, Tesh RB, Vinetz JM. Detection of pathogenic Leptospira spp. infections among mammals captured in the Peruvian Amazon basin region. Am J Trop Med Hyg. 2000;63:255-8.

17. Bessa TA, Spichler A, Chapola EG, Husch AC, de Almeida MF, Sodré MM, et al. The contribution of bats to leptospirosis transmission in São Paulo city, Brazil. Am J Trop Med Hyg. 2010;82:315-7. http://dx.doi.org/10.4269/ ajtmh.2010.09-0227 